\title{
The Challenges of Creativity in Software Organizations
}

\author{
Frank Ulrich $^{1}$ and Shegaw Anagaw Mengiste ${ }^{2}$ \\ ${ }^{1}$ Department of Computer Science, Aalborg University, Denmark \\ frank@cs.aau.dk \\ ${ }^{2}$ IT University of Copenhagen, Denmark \\ sanmaitu.dk
}

\begin{abstract}
Managing creativity has proven to be one of the most important drivers in software development and use. The continuous changing market environment drives companies like Google, SAS Institute and LEGO to focus on creativity as an increasing necessity when competing through sustained innovations. However, creativity in the information systems (IS) environment is a challenge for most organizations that is primarily caused by not knowing how to strategize creative processes in relation to IS strategies, thus, causing companies to act ad hoc in their creative endeavors. In this paper, we address the organizational challenges of creativity in software organizations. Grounded in a previous literature review and a rigorous selection process, we identify and present a model of seven important factors for creativity in software organizations. From these factors, we identify 21 challenges that software organizations experience when embarking on creative endeavors and transfer them into a comprehensive framework. Using an interpretive research study, we further study the framework by analyzing how the challenges are integrated in 27 software organizations. Practitioners can use this study to gain a deeper understanding of creativity in their own business while researchers can use the framework to gain insight while conducting interpretive field studies of managing creativity.
\end{abstract}

\section{$1 \quad$ Introduction}

Creativity has inherently become an important part of software development and use because the creative mindset is the pre-requirement in any innovation effort [23]. As Florida and Goodnight [19;131] noted: "The creative economy is here to stay, and companies that figure out how to manage for creativity will have a crucial advantage in the ever-increasing competition for global talent". Successful IS development companies such as Google and SAS have incorporated creativity as an important part of their strategies to create lasting innovations by creating creative environments, clear incentives for their employees, and a culture of social creativity where the contributions from each employee become important [19, 30]. Companies like LEGO have successfully created a business model that involves creative interactions with their customers through collaborative virtual worlds and online communities [45]. However, as Couger $[15 ; 230]$ argued, creativity is often treated as a "dangerous opportunity" that requires leadership and willingness to take risks. Hence, creative endeavors 
are not always equal to massive success, and organizations will often experience organizational challenges when embarking on creative endeavors [15].

To understand the challenges of managing creativity in a software setting, we define software organizations as information systems (IS) organizations, information systems development (ISD) organizations, and clone organizations (clone). In this paper, we treat IS organizations as those that use IT as a strategic driver for business operations and must be creative when implementing existing technologies, exploring new business opportunities, and enhancing their existing business in a competitive market [15]. ISD organizations are those that develop IT for others and must be creative when designing and creating innovative products or services [13]. Hence, IS and ISD organizations use time and effort in creating novel ideas for new market innovations to strategize their efforts for optimizing organizational performance, gaining competitive advantages, and attracting talented employees [19]. In between IS and ISD organizations, there are clone organizations. These are a mix between the IS and ISD organization because they develop IT as ISDs, and at the same time use this IT for internal and external business operations as an IS organization would. Regardless of the typology, however, software organizations are driven by continuous innovation to obtain and maintain their competitive advantages. As Couger clearly indicated, creativity has proven to be an important driver for IS, ISD, and clone organizations [15]. Moreover, creativity enhancing software has already proved useful for companies that are unrelated to IS or ISDs. As Rose [48] explained, creativity enhancing software have been developed for businesses in the areas of art, medicine, and chemistry. Likewise, creativity techniques originally developed for non-IS development have proven useful when software organizations are developing or exploring novel ideas for innovative software [15].

We wrote this paper upon a previous literature review [40]. This paper is a contingency of the original review where we address the subject of managing creativity challenges in software organizations. This paper differs from the original review by including literature we excluded from the original review. We examined the literature through a systematic process and provided interpretations to create a new framework of creativity management in software organizations. Moreover, we supplemented the literature with practical experiences collected through multiple empirical sources.

We structured the paper as follows: We initially provide a short research perspective for the study. Then we explain our research strategy, collection methods, and analysis. Next, we provide initial data analysis and describe in detail 21 challenges that software organizations experience when embarking on creative endeavors. We divide these challenges into seven different factors of creativity management and add our empirical findings. Finally, we collect our findings into a comprehensive framework for software organizations and explain how our study can help research and be deployed into practice.

\section{Research Perspective}

Understanding creativity in software organizations has since the early 1990's been a subject of IS research. This research surrounds the understanding of human-computer interaction [49, 59], proper training of individuals or groups [16], and techniques for 
facilitating creativity in the organizational setting $[14,15]$. Moreover, the organizational environment's influence over creativity $[5,15]$ and creating working strategies for creativity [61] have also proven important when enhancing creativity in software organizations.

Rhodes [46] suggested a framework of four interconnected components influencing creativity. These consist of press (environment), person (individuals and groups), product (creative results), and process (training and management) - each explaining aspects of creativity in the organizational context. We follow Rhodes' [46] track by examining a similar framework of interconnected factors and their underlying challenges.

The general consensus on creativity is that it is individuals' or groups' ability to come up with novel and useful ideas or solutions to problem [3, 4, 14, 15]. However, there is somewhat of a misconception of what a creative individual or group is in a software setting. Innovative software individuals or teams are typically viewed as either software designers or system developers [6, 13, 31]. Nonetheless, creativity in IS or ISDs is a conjoined effort by the entire organization and consists of many different factors and roles beyond the traditional software designer or system developers, as technology users can be equally creative [42]. Creative software individuals or groups can consist of customers, system developers, software designers, business analysts, portfolio managers, marketing managers, web designers, university researchers, computer and traditional artists, sourcing experts, project managers, CIOs, security experts, network analysts, customers, users, SPI specialists, etc. They all contribute with their own creative ideas for innovations to happen in the software organization, and they all use different practices, where creativity is needed and beneficial. Each profile must be taken into consideration when exploring the challenges of creativity in the software organization. Hence, our perspective on creativity management is the ability to understand the creative potential of the entire organization, by knowing how each organizational factor contributes to creating novel ideas, to identify them, to implement them, and finally to commercialize them into working innovations. This paper revolves around these factors of organizational creativity by examining those challenges that software organizations experience when they embark on creative endeavors. As such, our research subject is focused on the organizational aspect of creativity and not a study of creativity in a specific software setting (e.g. [63]. Hence, our research approach is guided by this overall question for inquiry: "What are the challenges of creativity in software organizations?"

\section{Research Approach}

To answer our research question, we used an adaptive multi-strategy approach [35] by providing interpretations from both quantitative and qualitative sources. According to Layder [35] the multi-strategy research approach offers several advantages over traditional research, as it increases the strength, density, and validity of the research by incorporating many data sources and strategies to approach the research subject, which enables the researcher to create more "robust" theoretical perspectives [35]. Hence, we divided the data collection between a survey in 27 software organizations and six interviews with employees of two of the organizations that participated in the survey. 
We constructed the survey around a cross-sectional research design [8, 54, 62], as the survey is consistent with more than one case and is fixed to a single point in time [8]. Moreover, we used a systematic and standardized approach for collecting data and examining relationships between variables [8]. This approach enabled us to view the integration of creativity management practices in software organizations and their relation to the literature reviewed by Müller and Ulrich [40].

For the interviews, we used an embedded case study design [62] by collecting data from different subunits in the organizations (e.g. departments, projects or management levels). This helped us to analyze different management perspectives in correlation to the challenges and factors from the literature.

Analytically, we worked interpretively both in our use of theory and in our data collection, whereby the theory and data played an equally vital role throughout the study when providing interpretations. For the interpretative approach, we used Walsham [55-57], who emphasized collecting and interpreting key concepts from the participants' social reality. However, when creating new interpretations, we analyzed all the data with a "healthy skepticism" [56]. This approach enabled us to support new ideas and concepts created during the analysis of the data provided by triangulating the findings [35] from the two data sources, which we transferred into a framework for creativity management at end of the paper. Moreover, these interpretations make the study highly inductive, as we locate management practices from the empirical data, rather than testing existing theoretical frameworks.

\subsection{Data Collection}

We collected the data through a survey of 31 managers and business analysts in 27 Danish software organizations. In addition, we conducted interviews with the CIO, a project manager, and a business analyst from the IT-department in a local Danish municipality, and the CIO, the head of innovation, and the head of product management in GameSim, a medium sized subunit of a much larger private and international organization. GameSim specializes in high tech computer game development for the private sector. The participants from the interviews also participated in the survey listed as cases 1-3 and 21-24 in Table 1.

Table 1. Participating organizations in the survey

\begin{tabular}{cccc}
\hline Case & $\begin{array}{c}\text { Organizational } \\
\text { size }\end{array}$ & $\begin{array}{c}\text { Public / private organiza- } \\
\text { tion }\end{array}$ & Type of organization \\
\hline $1-3$ & Medium & Private & Computer game developer (same \\
& & Public & organization) \\
4 & Very large & Public & Local municipality \\
5 & Very large & Public & Government organity \\
6 & Very large & Private & E-learning software developer \\
7 & Medium & Public & University college \\
8 & Very large & Private & AV equipment distributor \\
9 & Medium & Public & Local municipality \\
10 & Very large & Public & Knowledge organization in IT- \\
11 & Medium & & research \\
& & Private & IT-infrastructure developer \\
12 & Very large & Private & Administration software developer \\
13 & Medium & &
\end{tabular}


Table 1. (Continued)

\begin{tabular}{cccc}
14 & Very large & Private & $\begin{array}{c}\text { Major international software and } \\
\text { hardware developer } \\
\text { International process equipment } \\
\text { engineering company }\end{array}$ \\
15 & Very large & Private & Medical device data systems develop- \\
& er \\
16 & Medium & Private & Medical devise developer \\
17 & Small & Private & Hardware and software reseller and \\
18 & Medium & Private & developer \\
& Local municipality \\
19 & Very large & Public & Major internet service provider \\
20 & Very large & Private & Local municipality (same organiza- \\
$21-$ & Very large & Public & tion) \\
24 & Private & Healthcare software developer \\
25 & Large & Public & Local municipality \\
26 & Very large & Private & E-learning software developer \\
27 & Small & Public & Government organization \\
28 & Very large & Public & University college \\
29 & Very large & Private & Major information systems developer \\
30 & Very large & Private & International developer of labeling \\
31 & Large & & equipment and software \\
\hline
\end{tabular}

Organizational size $=$ Small (1-9 employees), Medium (10-99 employees), Large (100-499 employees), Very Large (501+ employees)

For conducting the survey, we used the social networking site Linkedin to locate participants. As Table 1 demonstrates, representatives from several types of software organizations chose to participate in the survey. In total, 38 chose to participate and complete the survey. However, we removed seven participants due to lack of credentials in the software sector or for not providing personal or company information.

We asked each participant to rate the integration of creativity management within the underlying challenges of the seven factors. In addition, participants were asked to provide information about their organization size (very large, large, medium, small), type of organization (private or public), management level they represent (CIO, project manager, or business analyst), IS use (developing for others, developing for themselves, or using IS developed by others). Moreover, we asked them if their organization uses IS to support creativity (use specialized IS, do not use IS for creativity, or use IS for creativity that is not designed for the purpose).

We asked each participant to rate the integration of creativity management within the underlying challenges of the seven factors. In addition, participants were asked to provide information about their organization size (very large, large, medium, small), type of organization (private or public), management level they represent (CIO, project manager, or business analyst), IS use (developing for others, developing for themselves, or using IS developed by others). Moreover, we asked them if their organization uses IS to support creativity (use specialized IS, do not use IS for creativity, or use IS for creativity that is not designed for the purpose).

\subsection{Data Analysis}

We analyzed the survey using SurveyXact, by conducting measurements on data frequency and the average distribution of data. Moreover, we calculated cross references 
between average distributions of data through Excel. We analyzed the survey results to establish casual relationships [54] between the factors of creativity and their challenges and the other groups of variables. These variables are organizational size, type of organization, management level, IS use, and use of creativity-enhancing software.

We analyzed the interviews using section transcription by providing in-depth notes for each interview [8]. We labeled each note with time, subject, and respondent, which provided a quick overview of the combined interview data. This approach enabled us to collect relevant key concepts when we interpreted and triangulated the survey and interview data.

\section{$4 \quad$ Mapping the Challenges}

To operationalize the challenges within the technology, environmental, institutional, individual, group, leadership, and external factors, we first identified each challenge by examining the existing literature. Second, we placed each challenge within a complementary factor. Specifically the work of several researchers [5, 13, 15, 19, 22] worked as guidelines, as they each provide a normative emphasis on the challenges of creativity in software organizations or provide emphasis on creativity challenges applicable to any organization regardless of internal structure or commercial output. However, most creativity challenges in the literature were descriptively operationalized by rigorously reviewing each challenge after reoccurrence in the literature and adding their normative guidelines to our in-depth description of each challenge. Finally, we connected the theoretical finding with our empirical findings. Hence, to clarify our observations from the survey and the interviews, we initially present our preliminary data findings in section 4.1 below. We then review the literature for each factor and connect our data findings in the subsequent sections for each factor.

\subsection{Preliminary Data Findings}

The survey elaborates how different managers in Danish software organizations have integrated the diverse challenges related to creativity in their organizations. The data collected in the survey demonstrates that the average integration score across the challenges is just below average. Their integration is aligned with the comments from survey and interview data. Our data demonstrated that Danish software organizations still face a range of challenges when integrating creativity management practices in their organizations. In addition, 45\% (14) of the managers and business developers participating were within public organizations and 55\% (17) were within private organizations. Moreover, there is little difference between private and public organizations when integrating the different creativity management challenges. The difference between the combined replies from private and public organizations is $3.6 \%$. Furthermore, when viewing employment among the participants, 23\% (7) of the participants were employed within top management as CEOs or CIOs, whereas, 64\% (20) were employed as mid-level managers such as project managers or department heads. Finally, $13 \%$ (4) were not employed within management, but were business developers. This finding demonstrates that the survey provided wide perspective within public and private software organizations. 
In the area of use and deployment of IS, 26\% (8) of the respondents answered that their organization developed IS for other organizations (ISD organizations). Also, $48 \%$ (15) deployed IS as a part of their daily business, but did not develop it themselves (ISD organizations), and 26\% (8) deployed IS as a part of their daily business and developed the systems themselves (clone organizations). Furthermore, clone organizations were $16.7 \%$ less likely to integrate management practices to deal with challenges in creativity than IS organizations, and equally $16.7 \%$ less likely to do it than ISD organizations. This finding demonstrates two different possibilities: ISD and IS organizations have a higher need for managing creativity; they are more likely to be driven by communities of creative practices as they are more engaged in creative behavior on a daily basis.

To our surprise, only one of the organizations had deployed specialized creativity support systems, and $29 \%$ (9) used IS for supporting creativity that were not designed for that purpose. In comparison, 65\% (20) did not use any form of IS driven creativity support. One did not know if their organization used it or not. The fact that the majority who used IS driven creativity support have chosen to use existing systems to support it is somewhat strange, because specialized systems could provide better possibilities. However, when interviewing the CIO of the local municipality the reason became apparent. As he stated:

"After an innovation management course we conducted for the managers in the municipality, we deployed a specialized tool to get people to work with idea development. However, people only partially used it because it was not a part of their daily workflow and they had to remember to use it. When people got back to work, they started to go back to use Outlook instead."

Hence, the reason for using existing systems to support creativity may be due to employees' and customers' preference to be creatively engaged in systems they use in their daily work activities.

\subsection{The Technology Factor}

Technology makes information and communication flow between stakeholders and is an important factor in organizational creativity [61]. When managers seek to increase organizational creativity, they can deploy computerized tools to support creative processes in organizations. Greene [24] demonstrated several characteristics of creativity software tools including: being exploratory in a sandbox mode; being engaged with content to advance learning and discovery; and being able to support collaboration, iterations, and challenging tasks. Creativity tools should also be domain-specific for the given task and make it easy for users to store, classify, relate, and retrieve things [24]. However, deploying or developing creativity tools or systems is not without challenges. When designing creativity tools or systems, developers must include the challenge of human-computer interaction by encapsulating user preferences, playfulness, interaction between design, and task-specific appliances [49, 59]. Hence, before development, business analysts must set design requirements that capture the organizational necessities and include business benefits from existing system portfolios through specific business plans [31, 58]. In addition, implementation of new IS requires user acceptance [18]. Users frame and make sense of technology differently 
within their own social and work-based arenas, which often collide with the implementation efforts [44, 60]. Thus, when implementing new IS (including creativity supported IS), managers must consider the social setting and users' preferences and take proper action when needed [20].

\section{Findings.}

Our findings showed that focus on human-computer interaction was less significant in organizations that develop creativity enhancing IS for themselves than in ISDs. Human-computer interaction was, however, slightly less integrated in clone and ISD organizations than IS organizations. Similar in technology acceptance, integration in clone organizations tends to be significant at a lower level than in ISDs, and somewhat lower than IS organizations. These findings demonstrate that ISD and IS organizations have a closer relationship to suppliers and customers and are more likely to collect management practice experiences and new system ideas when implementing and developing new systems. Another explanation can be a closer interaction with their business and an increased knowledge of organizational management practices when developing IS for creativity enhancement. Moreover, IS and ISD organizations prefer to implement creativity enhancement within their existing IT-portfolio. This increases technology acceptance, as employees and customers are more likely to use creativity enhancing software when it is included in systems they know and use.

\subsection{The Environmental Factor}

The environment in software organizations influences the creative output by providing social spaces for creative individuals and teams, which enables them to be inspired and construct ideas [14]. The physical environment in organizations have proven to have an impact on creativity efforts when developing new IS [5, 15]. Likewise, substantial evidence indicates that virtual environments in creativity enhancing software increases organizational creativity by creating social spaces for developers to share thoughts, find inspiration, compile relevant information, and conjoin creative efforts $[1,2,25,29,32-34,37,39,41,50]$. Moreover, when organizations are embarking on creative endeavors, managers must be aware of existing institutional practices and cultural norms that could create barriers for organizational creativity [15, 36]. Hence, they must create an organizational culture that supports creativity through direct strategies and leadership [5, 61].

\section{Findings.}

Our findings demonstrated equal medium integration in IS, ISD, and clone organizations when creating physical and virtual environments for creativity. For physical environment, some participants in the survey stated that their organization lacked proper equipment and facilities, which decreased creative thinking. When interviewing the business developer in the municipality it was also apparent that they had the proper equipment for enhancing creative thinking. However, the technology had technical difficulties, and they lacked training: 
"We have this smart board as a part of a test for the schools, but we primarily use it as an overhead projector... It is not properly calibrated... It is simply faster to do it with pen and paper and then take a picture and send it by mail... The school teachers are much better to use it than we are... the learning curve is simply too high."

This problem is also apparent with virtual environments. Most organization uses ana$\log$ devices, such as group brainstorming and pen and paper techniques, instead of software based solutions. However, some organizations use online idea boards in existing knowledge management systems. Here, employees share new ideas or save them for later use.

Private organizations are more likely than public organizations to create social environments for creativity. However, Danish public organizations are only just beginning to think in creativity and innovation, due to new policies for the public sector. Hence, they must continuously reform their business to save money while keeping or increasing the overall service level. Private organizations, however, have done this for years to gain competitive advantages. As the CIO of the municipality explained, they focused increasingly on creativity and innovation. To achieve their goals, leaders from the schools, nursing homes, and all other business areas of the municipality had to undergo innovation training and were included in the overall innovation efforts.

\subsection{The Institutional Factor}

Institutional barriers include negative perceptions towards new ideas and creative employees, not sharing relevant data, autocratic leadership, lack of task support, and fear of the unknown in creative processes, all of which negatively affect the outcome of organizational creativity $[9,15]$. Also, employees can have different creative styles, which place barriers on an individual level [15]. To reduce institutional barriers, managers must understand their employees' creative styles and create change-strategies for removing institutional barriers that poses negative influence on the creative output [15]. Moreover, Cooper [13] argued that creativity requires proper structures to be successful. Clear development goals reduce misplaced efforts by employees and groups and encourage productivity. However, tight control over creative processes will reduce autonomy and motivation of employees and negatively affect the creative outcome [9]. Managers must seek a balance between structure and over-the-edge autonomy ([13]. Agile development techniques such as extreme programming (XP) and the dynamic systems development method (DSDM) has proven to capitalize on employees' strengths [11], and give goals and constraints that provide boundaries for creativity to flourish instead for running amok [28]. However, creative endeavors in organizations are not without costs. Training and deployment strategies within the physical and virtual environment requires allocation of resources when enhancing the creative abilities in employees and leaders $[5,14,15]$.

\section{Findings.}

Institutional barriers still provide a massive problem by preventing creative people from being creative and exploring their potentials. Our findings revealed that both in the municipality and GameSim, many barriers were related to working in top-down 
governed organizations. These included strong expectations to get returns on investments, lack of possibilities to experiment without providing strong business cases for support of projects, lack of time to be creative, political trends influencing decision-making, expectations of continuous growth and system functionality, and lack of creative visioning in top management. However, this was much more apparent in GameSim, where traditional top management dictated the development strategies of the sub-unit. There was a better linkage in the municipality between the ITdepartment and the political leadership, which provided an increased ownership over projects and opened opportunities for experimentation without requirements of return of investment.

Defining structures for creativity is equally important. As one participant from a private developer in the survey stated:

"The waterfall model is our approach to project management ... This kills the creativity in the projects. The employees fight against this, which equal positive results. However, this process is very ad hoc when we have the time and resources."

Development processes in GameSim were equally very ad hoc. The creative process would stop when the requirement specifications were defined for a given project. In the municipality, they acted differently by strategizing projects as implementations projects. Requirements were either set in advance or defined as "next practice projects". Next practice projects included continuous creativity and experimentation during the entire project life cycle to provide more benefits and increase organizational knowledge in new areas. Hence, availability to experimentation is a valuable resource when embarking on creative projects. In GameSim, they created a system due to a larger certification. However, there were loosely defined requirements for the system, and government funding provided the proper time and resources for conducting the project. This provided GameSim room for developing new ideas by continuously interacting with their customers over a two-year period. The result was a very innovative product that both GameSim and their customers benefited greatly from.

\subsection{The Individual Factor}

To increase the creative output of the organization, employees require continuous education for developing their creative skills [16]. IS organizations also thrive from the individual's ability to be creative and create new ideas for continuous innovation ([19]. However, several factors influence individual impact on creative output. Creative employees are especially known for being exceptionally difficult to manage. They often know their own worth, have expert knowledge, and have A-type personalities and behavior that often results in conflicts with authorities [19, 22]. Managers must create an environment that not only empowers creative employees, but also attracts them to the organization [9].

\section{Findings.}

There is low overall integration in the challenges connected to the individual factor in comparison to the challenges connected to the other six factors, which indicates that the participating organizations have little emphasis on training their employees in 
creativity and manage creative people. However, our analysis showed many different approaches to provide training for creative employees. Some organizations would provide individual courses for employees, while others would hire external consultants for enhancing the overall creativity and innovation ability in the organization. Some would not provide any training at all and would rely on their employees' natural ability to be creative. However, training in creativity provides many benefits for organizations. In the municipality, a creativity and innovation workshop helped the employees to think differently and engage in experimentation, which provided solutions for a range of problems they faced.

Equally, in the daily management of employees, empowerment was important. Where the municipality used empowerment to encourage creativity for the employees, employees in GameSim were already empowered. The employees engaged in the creative development were some of the few specialists within their field. As the head of product development argued:

"Our employees are small kings within their own field and there is not a lot of team spirit”.

Hence, the challenge in GameSim was not to enable creative empowerment in their workforce, but to control the highly creative employees, as the individuality of these specialists often collided with the needs of the customers. Consequently, one of the job specifications for the head of product development was to enable communication between customers and the creative employees to provide encouragement for the employees and to insure business value to the customers from their new ideas.

\subsection{The Group Factor}

Creative activities are often conducted in teams. As Cooper [13] argued, IT-specific knowledge is an important capability in development teams. It enables them to act creatively according to the organizational context. Professional differences in development teams are thus important for the composition of development teams and overall success of projects [13]. Moreover, when giving tasks to creative individuals or creating creative teams, an important consideration is that individuals are different in their cognitive abilities, knowledge, behavior, and personality [5]. Equally important is the demographic, cultural, and social composition. Creative development teams perform better when they are composed of employees with different experience and education levels $[5,13,21,53]$. However, teams are less creative when they are comprised of employees with different social or cultural backgrounds, or different sexes [52]. Managers must understand these factors and their employees when they create development teams composed of individuals with different professional, social, and cultural backgrounds $[5,13,52]$. When teams are comprised of employees with different backgrounds and experiences, they require a higher level of communication and interaction to collaborate. As Cockburn and Highsmith [11;132] argued "Interestingly, people working together with good communication and interaction can operate at noticeably higher levels than when they use their individual talent". Hence, managers are required to use increased time and effort to create working development teams and to facilitate mediated communication and interaction in the teams [52]. 
Software organizations are also an entity of different communities of practices that influence organizational culture and norms, which results in learning and innovation, defines organizational structures, and creates institutionalization of those practices [7]. When creating creative groups, communities of practices are important for transferring organizational knowledge from individual to individual [15, 38]. In managing for creativity, managers must understand the existing communities of practices in the organization and actively use these in the knowledge creation processes, when changing culture and norms, and when transferring knowledge to create a shared understanding of problems.

\section{Findings.}

Creativity workshops can do a lot when attempting to increase organizational creativity. During a creativity and innovation workshop in the municipality, leaders across the organization were divided into groups. The purpose of the workshop was to place the participants out of their normal comfort zone and encourage them to think in digital solutions for their everyday problems. During the workshop, the leaders would then change groups and further develop the problems within a new group of people. This approach of constantly changing the leaders' environment broke down cultural differences and enabled the leaders to think differently, which resulted in novel ideas for digitalizing problems across the organization. Moreover, the survey showed that IS and ISD organizations have a similar integration of communities of practices, while clone organizations had a lower integration. This cause of difference can be related to a higher inclination in IS and ISD organizations to institutionalize existing practices through software use and development (e.g., [43, 44]).

The importance of mediated communication is also apparent. Without proper communication between team members and between departments, actors will lose important information that they can use as a source of inspiration of novel ideas. In both the municipality and GameSim they solved this problem by using IS for mediating the communication and information sharing process. GameSim used an intranet solution for their developers and business analysts, which contained design and development ideas and other relevant information provided by the employees. The municipality, alternatively, used an open intranet solution that allowed departments across the organization to view detailed information from digitalization projects in other departments.

\subsection{The Leadership Factor}

Managers' leadership abilities in software organizations are an important and often overlooked factor in the organizations' goal of increased creativity [19]. Managing IS does not only include delegating tasks and resources or creating of business plans for projects. IT also requires clear leadership, which involves individual human empathy and empowerment of employees $[5,9,15]$, and a clear understand of the group dynamics in development teams [13, 38]. Managers must be willing to take risks when needed $[9,15,51]$. Fear of failure in top management discourages employees and decreases the flow of novel ideas [15]. Moreover, managers must deploy creative techniques when needed and create incentives and motivation for employees and 
groups to enhance the creative output [14,15], while being able to include employees' personal characteristics when transferring techniques to system use [10]. Hence, managers must strategize and conceptualize the creative endeavors in IS organizations with the existing business processes, by providing plans for implementation of systems and change, training, evaluation, and diffusion of creativity tools and techniques $[14,15]$.

\section{Findings.}

The integration of incentives for creative development was the lowest of all the challenges despite it being a powerful motivator for increasing organizational creativity [15]. In our survey, $48 \%$ (15) answered that this challenge was not very well integrated in their organization, and only $6 \%$ (2) replied that it was very highly integrated. Hence, these software organizations lack a leadership culture that provides incentives. However, the findings from the interviews demonstrate a positive culture for empowerment, as employees and managers are encouraged to take ownership over projects.

Our findings also showed that there is some kind willingness to take risks with new ideas in software organizations. However, private organizations had a substantial higher integration than public organizations when it came to practices in risk-taking. The cause of this difference can come from Danish public organizations being subject to government laws, which prevent them for hiding their failures. This exposure to their customers' opinions (politicians and citizens) can make them less inclined to take risks, which affects their overall ability to engage in high-risk creative projects.

Deploying creativity techniques IS and ISD organizations were mostly on ad hoc initiatives. In the municipality, they would hire outside consultants to conduct their workshops, and when asked about the techniques deployed, they would only provide sporadic information about them. In GameSim, the same problem was apparent. The managers utilized creativity techniques such as brainstorming, but they had no defined approaches or predefined knowledge for deploying different creativity techniques.

The integration of strategizing creativity in the organizations was almost equally below average in both private and public organizations. The interviews somewhat explained this finding. In the municipality, they had only recently created a strategy for creativity and innovation one year prior to this study. In GameSim, a similar strategy was created only four months prior. However, this finding also indicates that both private and public organizations increasingly attempts to integrate creativity management practices in their organizations by defining overall strategies for creativity and innovation.

\subsection{The External Factor}

User and customer involvement are an important factor in system development [17]. As customer do not always know their needs, it is important to understand the user to make requirements [6]. Hence, for new business opportunities to emerge, novel ideas must be evaluated and screened before implementation [15]. Florida and Goodnight [19] recommended locating a balance between creating novel ideas and allocating customer needs through relationship building and testing schemes. However, analyzing customer needs in creative processes requires a culture of constructive criticism to 
deliver continuous quality [19]. This also requires a culture that emphasizes that some projects just fail. Organizations who punish employees who fail with projects will remove any incentives to be creative [5].

Using quality assurance schemes such as Software Process Improvement (SPI), Lean, and Six Sigma are common practices in software development to provide product and service quality and standardization of business processes for continuous customer value $[12,27]$. However, the discipline aspect of quality assurance schemes have been criticized for inhibiting creativity [47]. Conradi and Fuggetta [12] and Herbold [26] argued that organizations should balance their quality assurance efforts between discipline and creativity, where discipline support inspections and standardization of business processes, and creativity and collaboration support engineering and software design.

\section{Findings.}

Data from the survey and interviews revealed that organizations' business primarily use business cases before and after software implementation to evaluate novel ideas. Business cases have the advantage of providing a quick determination of business value for a given idea (e.g. [58]). In the two organizations in which we conducted interviews, they measured the quality of new ideas by level of business value. They would rarely use strict management methods, such as Lean, Six Sigma, and SPI, to ensure quality control of new ideas, which could be the cause of low integration in creating quality assurance schemes. Instead, their employees was encouraged or simply told to determine business value for each new idea before implementation. However, in the municipality, they would follow up on the business cases during implementation. This ensured that new ideas could emerge and add additional business value over the project's life cycle.

In most of the software organizations, user and customer involvement was important to insure business value for creative ideas. However, user involvements in the private organizations were significantly higher than in public organizations. In the municipality, the CIO agreed that they were good in involving their own users and suppliers in new digitalization project. However, they lacked a clear emphasis on involving their customers (the citizens). In GameSim, the customers were highly involved in the creation of new products, as they were a source of novel ideas during the requirements specification of new products and services. Moreover, the survey demonstrated a clear contrast to the overall integration of user and customer involvement between ISD, clone, and IS organizations. ISDs were far better at integrating user and customer involvement than IS organizations and somewhat better than clone organizations.

\section{$5 \quad$ A Framework for Creativity in Software Organizations}

From our synthesis of the literature and the empirical evidence, we constructed a framework consisting of the seven identified factors for creativity in software organizations. Moreover, we identified 21 challenges in creativity management within the seven factors.

The schematic representation of our proposed framework is presented in in Figure 1 and demonstrates how multiple challenges can unfold themselves in software 
organizations. These challenges exist within seven factors in the creative context of organizational creativity. The framework also demonstrates the mutual influence from each of the seven factors on a software organizations creative output (ideas generation). Each factor has individual challenges attached that are illustrated with unbroken lines between the challenges and their factors. The challenges define the factors, which in return affects the creative context and ultimately the creative output of software organizations. For example, a challenge can be the demographic composition of creative development teams in the group factor, or the challenge of organizational barriers in the institutional factor.

Inspired by Rhodes' (1961) thinking, no individual factor of creativity stands alone, but all are connected in unity. Hence, the different factors can impose influence over other factors. For example, creativity support systems can influence the creativity in individuals and groups, while individuals can affect how groups function. Moreover, leadership influences the creative environment, while external factors like idea evaluation schemes and relationships with customers can influence the institutional structures of the organization. Hence, this area of influence represented in the middle of Figure 1, demonstrating how each factor influences the organizational context through interaction with other factors. This interaction between factors also defines the creative context of the organization. As illustrated, this mutual influence and interaction between different factors ultimately outlines the creative output of the software organization.

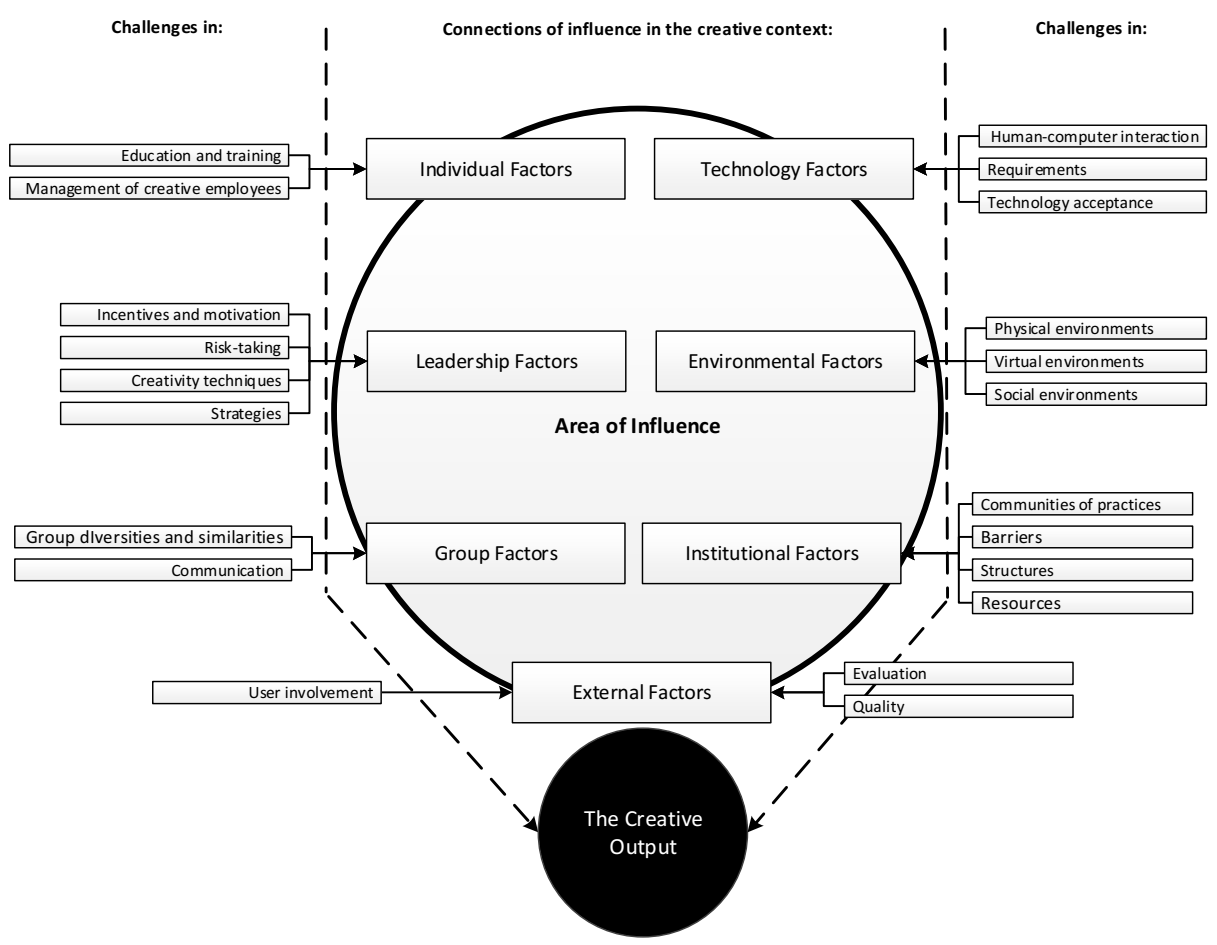

Fig. 1. A framework of creativity challenges in software organizations 


\section{Conclusion}

Creativity management has become an increasingly important factor in conducting product and service innovation in software organizations. Creativity management involves increasing expertise in techniques, leadership, strategies, evaluation, and human-computer interaction in order to break down management challenges and achieve the necessary benefits of organizational creativity initiatives.

In this paper, we asked ourselves the following research question: "Which challenges of creativity does software organizations experience?" To this end, we created a framework that influences creativity management in software organizations. We identified seven organizational factors of the technology, environment, institution, individuals, groups, leadership, and external issues. Moreover, we identified $21 \mathrm{com}$ mon challenges within the different factors and provided a combined framework with a comprehensive theoretical foundation and an interpretive research study to support it, which demonstrates how managers in software organizations practice creativity management. The framework also shows that organizational creativity is a combined entity consistent of different factors, which impose mutual influence on each other. Managers and researchers must be aware of this mutual influence and are encouraged to provide new initiatives for practice and research that support organizational creativity as a combined entity.

The research provides several insights for creativity management in technologydriven organization. For example, our results showed that managers or employees often conducted creativity in practice through ad hoc initiatives rather than doing it in a structured form. However, the study also provided valuable insights into creativity management practices that are transferrable to normative guidelines for creativity enhancing software that practitioners could benefit from. As such, it became clear that creativity enhancing software requires a number of different features, including integration in the existing IS-portfolio, integration of knowledge management systems, and information awareness, design usability and training, mediated communication, pre-selection of tools, empowerment through incentives, and computerized evaluation of new and novel ideas. In addition, it requires additional management practices including clear strategies for creativity and innovation before implementation, culture building, and empowerment trough ownership of projects and freedom for experimentation. Such experimentation must include allocation of the necessary resources and clear leadership from top management when taking new risks.

However, when writing this paper, we also realized that creativity encompass a far wider complexity across a software organization than this paper is able to present. Hence, researchers can use our findings to gain a better understanding of how these factors and challenges are interconnected and influence the complex and often chaotic endeavor of creating novel and useful ideas for new software innovations.

\section{References}

1. Abrams, S., et al.: QSketcher: An environment for composing music for film (2002)

2. Aiken, M., Carlisle, J.: An automated idea consolidation tool for computer supported cooperative work. Inf. Manag. 23(6), 373-382 (1992) 
3. Amabile, T., et al.: Affect and creativity at work. Adm. Sci. Q. 50, 367-403 (2005)

4. Amabile, T.M., et al.: Assessing the work environment for creativity. Acad. Manag. J. 39(5), 1154-1184 (1996)

5. Amabile, T.M.: How to kill creativity. Harv. Bus. Rev. 76(5), 76-87 (1998)

6. Beyer, H., Holtzblatt, K.: Contextual Design - Defining customer-centered systems. Academic Press, London (1998)

7. Brown, J.S., Duguid, P.: Organizational learning and communities-of-practice: toward a unified view of working, learning, and innovation. Organ. Sci. 2(1), 40-57 (1991)

8. Bryman, A.: Social Research Methods. Oxford University Press, New York (2004)

9. Catmull, E.: How Pixar Fosters collective creativity. Harv. Bus. Rev. 86(9), 64-72 (2008)

10. Cheung, P.K., et al.: Does knowledge reuse make a creative person more creative? Decis. Support Syst. 45(2), 219-227 (2008)

11. Cockburn, A., Highsmith, J.: Agile Software Development: The People Factor. Softw. Manag. 34(11), 131-133 (2001)

12. Conradi, R., Fuggetta, A.: Improving software process improvement. IEEE Softw. 19(4), 92-+ (2002)

13. Cooper, R.: Information technology development creativity: A case study of attempted radical change. MIS Q. 24(2), 245-276 (2000)

14. Couger, J., et al.: (Un)structured creativity in information systems organizations. MIS Q. 17(4), 375-397 (1993)

15. Couger, J.D.: Creativity \& Innovation in information systems organizations. Boyd \& Fraser, Danvers (1996)

16. Couger, J.D.: Creativity: Important addition to national joint undergraduate IS curriculum. J. Comput. Inf. Syst. 37(1), 39-41 (1996)

17. Davenport, T.H.: Process Innovation - Reengineering work through information technology. Havard Buisness School Press, Boston (1993)

18. Davis, F.D.: Perceived usefulness, perceived ease of use, and user acceptance of information technology. Mis Q. 13(3), 319-340 (1989)

19. Florida, R., Goodnight, J.: Managing for Creativity. Harv. Bus. Rev. 83(7-8), 124-131 (2005)

20. Gallivan, M.J.: The influence of software developers' creative style on their attitudes to and assimilation of a software process innovation. Inf. Manag. 40(5), 443-465 (2003)

21. Gero, J.S.: Computational models of creative designing based on situated cognition (2002)

22. Goffee, R., Jones, G.: Leading clever people. Harv. Bus. Rev. 85(3), 72-+ (2007)

23. Govindarajan, V., Trimble, C.: The Other Side of Innovation: Solving the Execution Challenge. Harvard Business School Publishing, Boston (2010)

24. Greene, S.L.: Characteristics of applications that support creativity. Commun. ACM 45(10), 100-104 (2002)

25. Hailpern, J., et al.: TEAM STORM: Demonstrating an interaction model for working with multiple ideas during creative group work. In: Proceedings of the 6th ACM SIGCHI Conference on Creativity \& Cognition, pp. 193-202. ACM, Washington, DC (2007)

26. Herbold, R.J.: Inside Microsoft - Balancing creativity and discipline. Harv. Bus. Rev. 80(1), 72-79 (2002)

27. Hicks, B.J.: Lean information management: Understanding and eliminating waste. Int. J. Inf. Manage. 27, 233-249 (2007)

28. Highsmith, J.: What Is Agile Software Development? J. Def. Softw. Eng. 15(10), 4-9 (2002)

29. Hori, K.: A system for aiding creative concept-formation. IEEE Trans. Syst. Man Cybern. 24(6), 882-894 (1994) 
30. Iyer, B., Davenport, T.H.: Reverse engineering Google's innovation machine. Harv. Bus. Rev. 86(4), 58-68 (2008)

31. Kappel, T.A., Rubenstein, A.H.: Creativity in design: The contribution of information technology. IEEE Trans. Eng. Manag. 46(2), 132-143 (1999)

32. Kerne, A., et al.: CombinFormation: Mixed-Initiative Composition of Image and Text Surrogates Promotes Information Discovery. Acm Trans. Inf. Syst. 27(1) (2009)

33. Kletke, M.G., et al.: Creativity in the organization: the role of individual creative problem solving and computer support. Int. J. Hum. Comput. Stud. 55(3), 217-237 (2001)

34. Kohler, T., et al.: Co-creation in virtual worlds: the design of the user experience. MIS Q. 35(3), 773-788 (2011)

35. Layder, D.: Sociological Practice - Linking theory and practice. Sage Publications Ltd., London (1998)

36. Leonardi, P.M.: Innovation Blindness: Culture, Frames, and Cross-Boundary Problem Construction in the Development of New Technology Concepts. Organ. Sci. 22(2), 347-369 (2011)

37. Maccrimmon, K.R., Wagner, C.: Stimulating ideas through creativity software. Manage. Sci. 40(11), 1514-1532 (1994)

38. Malhotra, A., et al.: Radical innovation without collocation: A case study at BoeingRocketdyne. Mis Q. 25(2), 229-249 (2001)

39. Massetti, B.: An empirical examination of the value of creativity support systems on idea generation. MIS Q. 20(1), 83-97 (1996)

40. Müller, S.D., Ulrich, F.: Creativity and Information Systems in a Hypercompetitive Environment: A Literature Review. Commun. Assoc. Inf. Syst. 32, 175-200 (2013)

41. Nakakoji, K., et al.: A framework that supports collective creativity in design using visual images (1999)

42. Nambisan, S., et al.: Organizational mechanisms for enhancing user innovation in information technology. Mis Q 23(3), 365-395 (1999)

43. Orlikowski, W.J.: The duality of technology - Rethinking the concept of technology in organizations. Organ. Sci. 3(3), 398-427 (1992)

44. Orlikowski, W.J.J., Gash, D.C.C.: Technological frames - Making sense of information technology in organizations. Acm Trans. Inf. Syst. 12(2), 174-207 (1994)

45. Piller, F., et al.: Overcoming mass confusion: Collaborative customer co-design in online communities. J. Comput. Commun. 10(4) (2005)

46. Rhodes, M.: An analysis of creativity. Phi Delta Kappan 42, 305-310 (1961)

47. Roger, W.H., Martha, M.G.: Lean Six Sigma, creativity, and innovation. Int. J. Lean Six Sigma. 1(1), 30-38 (2010)

48. Rose, J.: Software Innovation - Eight work-style heuristics for creative system developers. Software Innovation, Aalborg (2011)

49. Shaw, T., et al.: The effects of computer-mediated interactivity on idea generation - An experimental investigation. Ieee Trans. Syst. Man Cybern. 23(3), 737-745 (1993)

50. Shneiderman, B.: Creativity support tools. Commun. Acm. 45(10), 116-120 (2002)

51. Sutton, R.I.: The weird rules of creativity. Harv. Bus. Rev. 79(8), 94-103 (2001)

52. Thatcher, S.M.B., Brown, S.A.: Individual creativity in teams: The importance of communication media mix. Decis. Support Syst. 49(3), 290-300 (2010)

53. Tiwana, A., McLean, E.R.: Expertise integration and creativity in information systems development. J. Manag. Inf. Syst. 22(1), 13-43 (2005)

54. De Vaus, D.: Research Design in Social Research. Sage Publications Ltd., London (2001)

55. Walsham, G.: Doing interpretive research. Eur. J. Inf. Syst. 15(3), 320-330 (2006) 
56. Walsham, G.: Interpreting Information Systems in Organizations. Wiley, Chichester (1993)

57. Walsham, G.: Interpretive case studies in IS research: Nature and method. Eur. J. Inf. Syst. 4(2), 74-81 (1995)

58. Ward, J., et al.: Building better business cases for IT investments. MIS Q. Exec. 7(1), 1-15 (2008)

59. Webster, J.: Microcomputer playfulness: Development of a measure with workplace implications. Mis Q 16(2), 201-226 (1992)

60. Weick, K.E., et al.: Organizing and the process of sensemaking. Organ. Sci. 16(4), 409-421 (2005)

61. Woodman, R.W., et al.: Toward a theory of organizational creativity. Acad. Manag. Rev. 18(2), 293-321 (1993)

62. Yin, R.K.: Case Study Research - Design and Methods. SAGE Publications Ltd., London (2003)

63. Aaen, I.: Essence: Facilitating software innovation. Eur. J. Inf. Syst. 17(5), 543-553 (2008) 